\title{
Die spezifische Behandlung der Tuberkulose mit Endotin.
}

Von

\section{Dr. Gabrilowitsch,}

Chefarzt zu Halila.

Die spezifische Behandlung der Tuberkulose besteht in der Anwendung von Tuberkelbazillenpräparaten, die auf das erkrankte Organ direkt wirken. Durch diesen Vorgang wird aber auch der Gysamtorganismus günstig beeinflusst, durch die Entlastung von giftigen Produkten.

Allein, es darf nicht ausser acht gelassen werden, dass diese Therapie nur dann zum Ziele führt, wenn sie in energischer urd zielbewusster Weise gehandhabt wird.

Der Schöpfer der spezifischen Behandlungsmethode, $\mathrm{R}$ ob e r $\mathbf{t}$ $\mathrm{K} o \mathrm{ch}$, hatte zuerst die Beweise geliefert, dass die Tuberkulose auf diesem Wege heilbar sei. Wenn auch seine Ansichten über das Wesen des bei diesem Prozesse sich abspielenden Vorgangs nicht von allen Ärzten geteilt werden, so können die Endresultate doch nicht hinweggeleugnet werden. Sein Verdienst ist es, wenn heute die spezifische Behandlung in der ganzen Welt Anhänger und Freunde gefunden. Dass aber alle bisherigen Leistungen noch lange nicht den realen Anforderungen genügen, versteht sich von selbst. Erst die eifrigste Beteiligung von seiten des Staates und der Ärzte würde die Sache kräftigst fördern und der Tuberkulose ihre Opfer entreissen.

$\mathrm{Zu}$ diesem Zwecke bedarf es freilich vor allen Dingen der Unterstützung der Ärzte. Solange aber in diesen Kreisen noch nicht die vollste Überzeugung Platz gegriffen, dass die spezifische 
Therapie die Tuberkulose erfolgreich bekämpfen könne, solange wird auch die Phthiseotherapie nur langsame Fortschritte machen in der Bekämpfung des Übels als Volkskrankheit.

Die frühere Stellung, welche die meisten Ärzte der spezifischen Behandlung gegenüber einnahmen, und welche noch gegenwärtig viele veranlasst, derselben im besten Falle eine Indifferenz entgegenzubringen, erklärt sich einmal aus den Misserfolgen der ersten Kochschen Ära, und zweitens aus gewissen Trugschlüssen, dassein Spezifikum gegen die Tuborkuloso jede Form derselben radikal heilen müsse.

Was den ersten Punkt betrifft, so steht es fest, dass die meisten Ärzte, die sich da ablehnend verhalten, nicht aus eigener Erfahrung sprechen, sondern sich auf Handbücher und andere Autoren berufen. Ich kann jedoch bezeugen - und mit mir wahrscheinlich noch viele andere -, dass nicht wenige von uns seinerzeit mit Tuberkulin behandelten Kranken bis heute gesund geblieben sind. Es sind also nicht alle gestorben, wie das so oft behauptet wird.

Der zweite Punkt ist noch weniger stichhaltig. Besitzt denn die Medizin überhaupt Mittel, die in solcher Weise wirken, d. h. jedesmal und bei jeder Form. Bei der Tuberkulose aber, wo die Vergiftungserscheinungen sich in erster Linie geltend machen, ganz unabhängig von der Krankheitsform, dürfen solche unmöglichen Forderungen nicht gestellt werden. Abgasehen von den in akuter Weise verlaufenden Prozessen haben auch chronische Formen oft schon einen solchen Grad der Erschöpfung des Gesamtorganismus bewirkt, dass eine Änderung des Zustandes unmöglich wird, ganz unabhängig von dem Grad der anatomischen Ausbreitung.

Meine ersten Erfahrungen mit Tuberkulin ( $\mathrm{o} \mathrm{o} \mathrm{h} \mathrm{scher} \mathrm{Lymph})$ ) sammelte ich in den Jahren 1890/1891, worüber ich seinerzəit berichtete (Wiener med. Wochenschr. 1891, Nr. 4). Zeitweise hatte ich die Tuberkulinbehandlung unterbrochen, um verschiedene andere Präparate zu versuchen. (Über das Antiphthisin Klebs, Wiener med. Wochenschr. 1895.)

Trotzdem, dass minimale Dosen injizieirt wurden $(0,0005 \mathrm{mg})$, wurden doch mit dem $\mathrm{Kochschen} \mathrm{Tuberkulin} \mathrm{sehr} \mathrm{bedeutende} \mathrm{All-}$ gemeinreaktionen ausgelöst. So konnte bei einer Leichtkranken, fieberfrei, nach der ersten Injektion eine rapide Steigerung der Körperwärme bis $39,5^{\circ}$ beobachtet werden, die 48 Stunden lang anhielt. Alle übrigen Reaktionserscheinungen verliefen äusserst stürmisch, das Allgemeinbefinden war und blieb schlecht, selbst nachdem die Temperatur schon normal geworden war. Bei den übrigen Kranken war zwar auch eine Temperatursteigerung zu beobachten, die aber 
fast nie über $39,0^{\circ}$ hinausging. Als weitere Symptome sind zu nennen: Schüttelfrost, Gliederschmerzen, Üblichkeit, Erbrechen (zuweilen mit Galle oder Blutbeimengungen).

Besonders auffallend war das Anschwellen der Lymphdrüsen in der Achselhöhle, ein Symptom, das sich auch beim Antiphthisin Klebs stets wiederholte.

Scharf ausgesprochene Herdreaktionen waren gleichfalls zu konstatieren; ja selbst Vergrösserungen der Dämpfungsbezirke über der Lunge.

Im Anschluss an alle genannten Erscheinungen war eine Körpergewichtsabnahme zu verzeichnen, wenigstens bald nach den Injektionen.

Immerhin muss ich zugeben, dass viela von den Kranken, die mit dem $\mathrm{Koch}$ schen Tuberkulin in der ersten Ära behandelt wurden, geheilt wurden; ja, dass einige bis jetzt noch am Leben sind.

Als nun in der späteren Zeit die ganze Behandlungsmethode eine Änderung erfuhr, die namentlich die Dosierung betraf, erschienen Berichte, die die Tuberkulinanwendung weniger gefährlich schilderten.

Meine Erfahrungen ergaben entschieden günstige Resultate, die aber stets auch von Reaktionserscheinungen begleitet waren. (Russky Wratsch. 1905. - Nowojew Medizine, 1908, Nr. 6.)

Mit dem Alttuberkulin war es ein Ding der Unmöglichkeit, sichere Anhaltspunkte zu finden, um die Reaktionserscheinungen zu, vermeiden. Bald waren es die leichtesten Formen, die eine Reaktion gaben, bald die schweren. Zuweilen glaubte man im Laufe der Behandlung die Überempfindlichkeit beseitigt, da kam eine heftige Reaktion. Arzt und Patient wurden nervös und mussten das Vertrauen zu dem Mittel verlieren.

Dass übrigens in weitesten Kreisen die Unmöglichkeit, so weiter zu behandeln, erkannt wurde, erhellt aus den Versuchen, eine passende Dosierung zu finden. Man beschäftigte sich weniger mit der Krankheitsform, denn das war ein schweres Kapitel, auch nicht mit dem Studium: des Präparates selbst, sondern man wählte den Weg, auf dem man am raschesten zum Ziele kommen musste - die Dosierung. Nun hat ja eine entsprechende Dosierung gewiss etwas für sich; es kann nicht bestritten werden, dass die Dosis zuweilen zu hoch war. Aber es lag die Gefahr nahe, dass man auf diesem Wege zu den unmöglichsten Dingen gelangen konnte; zu Dosierungen, die sich unseren Begriffen ganz entziehen. Wenn nun selbst bei den minimalsten Dosen unerwartete Reaktionen auftreten, wenn die Lungenkrankheit an sich niemals massgebend ist, um eine richtige 
Dosierung zu gewährleisten, so bleibt nur die dritte Möglichkeit, dass wir das Präparat selbst genauer studieren müssen.

Die wirksame Substanz im Tuberkulin ist ein Ferment, das die Herdreaktion bewirkt, und zur Resorption des tuberkulösen Gewebes führt oder zu seiner Einschmelzung (cf. Gabrilowitsch Vortrag, Brüsseler Konferenz, 1910). Als nächste Folge sind zu verzeichnen: Hyperämie und thermale Temperatursteigerung. Dieses Ferment, das ich mit dem Namen „Endotin“ bezeichnet habe, wird unter natürlichen Verhältnissen namentlich von älteren Tuberkelbazillen in ausreichender Menge geliefert, um das tuberkulöse Gewebe einzuschmelzen. So entsteht die Höhle mit einem Ausführungskanal (Bronchus), der die Zerfallsprodukte nach aussen abführt. Die Höhle reinigt sich, die Wände schrumpfen und der Kranke gesundet. Die künstliche Einführung des Endotins bezweckt nur, den Einschmelzungsprozess zu unterstützen. Da nun erwiesenermassen das Endotin keine Re aktionserscheinungen macht, im übrigen aber den lokalen Prozess sehr rasch bessert, so benutze ich jetzt das Präparat ausschiessllich zur Behandlung der Tuberkulose. Ich benutze es in allen drei Stadien der Krankheit; auch bei Fiebernden. Ich beschränke die Behandlung nicht.auf bestimmte Kategorien, sondern benutze es überall da, wo der Allgemeinzustand befriedigend ist. Auch Kinder behandle ich mit dem Endotin, sobald klinische Symptome da sind, als: schlechte Ernährung, die sich mit den üblichen Behandlungsmethoden nicht bessern lässt; subfebrile Temperatur. steigerung, Lymphdrüsenschwellungen, oder geringfügig» Veränderungen im Lungengewebe.

Das Mittel ist nie kontraindiziert, es sei denn, es handle sich um Sterbende.

Ich beginne mit $0,01 \mathrm{mg}$. Eine Herdreaktion ist nicht nur nicht schädlich, sondern ich halte sie für wünschenswert, um eine erfolgreiche Kur zu erzielen. Bei der Endotinbehandlung sind hohe Reaktionen und Tuberkulinschäden gar nicht zu beobachten. Eine Kur dauert unter normalen Verhältnissen $6-8$ Wochen. Ich dosiere folgendermassen: Die Dosis wird solange verdoppelt, bis die Herdreaktion sich einstellt. Von da ab wird langsamer vorgegangen oder die Kur zum Abschluss gebracht, wenn die katarrhalischen Erscheinungen in der Lunge ganz geschwunden sind. In den meisten Fällen schliesse ich jedoch die Kur mit $100 \mathrm{mg}$.

Die Injektionen werden am Morgen gemacht und meist die Skapulargegend für die Injektion benutzt.

Die hygienisch-diätetische Behandlung ist wünschenswert bei der Kur, notwendig ist sie jedoch nicht, wie die Erfolge der ambulantan Behandlung: bewiesen haben. 
Eine Wiederholung der Kur muss vorgenommen werden, sobald die katarrhalischen Erscheinungen sich wieder einstellen oder noch nicht ganz geschwunden sind.

Statistische Angaben sind weiter unten angeführt.

Während der 'Tuberkulintherapie verwende ich keine anderen spezifischen Mittel.

Das Alttu berkulin ist bekanntlich ein Glyzerinextrakt aus den Reinkulturen der Tuberkelbazillen und wird für die spezifische Behandlung benutzt. All die vielen anderen Präparate sind nichts anderes als Alttuberkulin.

K.o c h formulierte seine Herstellungsweise folgendermassen: „In das einfache Extrakt gehen aus den Tuberkelbazillen natürlich neben der wirksamen Substanz auch die übrigen, in 50\% Glyzerin löslichen Stoffe über, und es finden sich darin eine gewisse Menge von Mineralsalzen, färbende Substanzen und andere unbekannte Extraktivstoffe. Einige dieser Stoffe lassen sich ziemlich leicht daraus entfernen". . . ,Auch die Farbstoffe lassen sich beseitigen. Für die Anwendung in der Praxis bietet diese Reinigung des Glyzerinextraktes indessen keine Vorteile, weil die so entfernten Stoffe für den menschlichen Organismus ind ifferent sind, und also der Reinigungsprozess das Mittel nur unnötigerweise verteuern würde."

Diese beiden Argumente schienen demnach massgebend, den Reinigungsprozess zu unterlassen. Das zweite Argument ist natürlich an und für sich belanglos, nicht so das erste. Die hauptsächlichsten Bestandteile des Alttuberkulins organischer Natur sind: Protalbumosen, Deuteroalbumosen, kleine Mengen ron Heteroalbumosen, Spuren von Dysalbumosen und Glyzerin.

Da die Tuberkelbazillen auf Nährböden Deuteroalbumosen aus Protalbumosen bilden können und dieselben, wio überhaupt Albuminate und Albumosen als solche, den tuberkulösen Tieren einverleibt, zu Temperatursteigerungen führen, so können diese Stoffe jedenfalls nicht zu den indifferenten gerechnet werden.

Subkutane Injektionen von $0,02 \mathrm{~g}$ Deuteroalbumosen verursachten beim Menschen wenig Unbehagen, erst bei 0,05 bis $0,07 \mathrm{~g}$ entstand beim gesunden Menschen Fieber; in zwei Fällen traten palpable Milztumoren auf. Mit Deuteroalbumosen konnten alle Tuberkulinreaktionen hervorgerufen werden, sowohl beim Menschen, als auch beim Tier. Es konnte nachgewiesen werden, dass die stärkere Tuberkulinwirkung bedingt ist durch dessen Gehalt an echtem Pepton, das immens giftig ist.

Es muss also daran festgehalten werden, dass in sämtlichen Tuberkulinen Stoffe enthalten sind, die an und für sich schon Fiebər 
erzeugen können; auf die Dosis kommt es gar nicht an, da die Dosis in jedem einzelnen Fall nicht vorher bestimmt werden kann.

War es aber notwendig, dem Organismus zu Heilzwecken das ganze Gemisch der Kultur zu injizieren? Diese Stellung zu der Frage musste sich billigerweise aufdrängen, wenn der Umstand in Betracht gezogen wurde, dass selbst indifferente Lösungen von Glyzerin, Kochsalz etc. bei subkutaner Anwendung Temperaturstaigerungen bis über $40^{\circ}$ bei tuberkulösen Meerschweinchen hervorrufen konnten.

Es drängte sich somit der Gedanke auf, ein Tuberkulin rarzustellen, das die von $\mathrm{K}$ och für indifferent gehaltenen Stoffe nicht enthielt, ohne die wirksame Substanz in irgend einer Weise zu verändern.

$\mathrm{K}$ o ch hatte die Meinung geäussert, dass die wirksame Substanz im Tuberkulin ein Derivat der Eiweisskörper sei, dass es diesen nahestehe, aber nicht zur Gruppe der sogenannten Toxalbumine gehöre, da sie hohe Temperaturen ertrage und im Dialysator leicht und schnell durch die Membran geht. „Das im Extrakt vorhandene Quantum der Substanz ist allem Anschein nach ein sehr geringes; ich schätze es auf Bruchteile eines Prozents. Wir würden es, wenn meine Voraussetzungen richtig sind, also mit einem Stoff zu tun haben, dessen Wirksamkeit auf tuberkulös erkrankte Organismen weit über das hinausgeht, was uns von den am stärksten wirkenden Arzneistoffen bekannt ist."

Dies alles liess sich bestätigen, vornehmlich auch, dass die wirksame Substanz sehr hohe Temperaturen erträgt.

Ich habe die wirksame Substanz als Endotin bezeichnet, zum Unterschied von jenen Körpern, die der Eliminierung unterlagen, also vornehmlich Eiweissstoffe und Fette, und die den gemeinsamen Namen $\mathbf{E k}$ dotine erhielten.

Das Endotin ist kein neues Tuberkulinpräparat, sondern Alttuberkulin, auf chemischem Wege verändert.

Das Endotin unterlag daher jedesmal der vorgeschriebenen Prüfung, da es aus dem in Deutschland staatlich geprüften Alttuberkulingewonnen wurde. Es hat demnach auch die Konstanz der Zusammensetzung und Wirkung des Ausgangsmaterials. Die chemische Bearbeitung kann natürlich an dieser Konstanz des Präparates michts ändern, w e il e in $\theta$ Behandlung mit Reagenzien in bestimmter Quantität und Konzentration stets dieselbe Wirkung a usüben muss. Die Reinigung muss allerdings so vorgenommen werden, dass die Vermischung des Originaltuberkulins mit dem be- 
treffenden Reagens eine sehr inmige ist. Es muss ausserdem genügend Zeit gelassen werden, um die Scheidung der Substanzen voneinander scharf durchzuführen. $Z u$ diesem Zwecke bedient man sich am besten des Scheidetrichters. In erster Linie wird Xylol zur Entfettung benutzt. Es folgen dann Chloroform und Äther. Besonders ist darauf zu achten, dass keine Spuren des Chloroforms zurückbleiben. Die Ätherausschüttelung ist in dieser Hinsicht von allergrösster Bedeutung. Nach der Xylol-, resp. Chloroformanwendung ist Erwärmen mit nachfolgendem Zentrifugieren notwendig. Die Ei. weisskörper werden dann leicht mit heisser verdünnter Lauge entfernt.

Der prinzipielle Unterschied zwischen Alttuberkulin und Endotin ist ersichtlich beim Vergleich ihrer chemischen Analysen. Aus dem physiologisch-chemischen Laboratorium der Universität Breslau liegen diese Analysen vor. Ich begnüge mich mit dem Anführen der Schlusssätze, da die sehr ausführlichen Berechnungen und Tabellen der Originalarbeit hier nicht in extenso wiedergegeben werden können.

„Es ist daher ersichtlich, dass das Quantum der im Endotin enthaltenen Eiweisskörper sehr gering ist, im Vergleich zu den im Alttuberkulin enthaltenen. .." „Beim Vergleich des mit Alkohol, Xylol, Chloroform und Äther bearbeiteten Tuberkulins mit dem Alttuberkulin muss geschlossen werden, idass in qualitativer Beziehung diese Bearbeitung wenig wirksam war, in quantitativer dagegen sehr bedeutend. Die relative Steigerung des Prozentsatzes an Amidosäuren allein zeigt schon, in welch beträchtlicher Weise die Zahl der stickstoffhaltigen Körper verringert ist. Der gänzlich unbedeutende Gehalt an ilbumosen tritt hier in erste Reiho. Das Quantum der Farbstoffe und Extraktivstoffe ist ebenfalls gering. Gehalt und Quantum der Aschenbestandteile ist fast unverändert."

Der Beweis für die nach der chemischen Behandlung des Präparates noch erhaltene spezifische Aktivität wurde durch den Tierversuch erbracht.

Eine Herde von jo Kühen wurde mit $0, \tilde{\partial} \mathrm{g}$ Bovotuberkulin Behring geimpft. Deutliche Reaktion zeigten 8 Kühe. Dieselbøn wurden isoliert und der Endotinbehandlung unterworfen. (cf. Stenius.)

Vor der Behandlung wurden die Kühe genau untersucht, sowohl in bezug auf das Allgemeinbefinden, als auch auf den Zustand der Lungen. Herdreaktion. Dieselbe bezieht sich vornehmlich auf Atmungsgeräusche. Sie werden als verstärkt bezzichnet; 
in einigen Fällen wurde unterdrücktes Atmen (abgeschwächtes) beobachtet. Ausserdem war verstärktes Rasseln zu notieren.

Kniefalten, Euter- und Maxillardrüsen schwollen unter der Behandlung an.

Nach der Behandlung waren sämtliche pathologische Erscheinungen geschwunden; ebenso der Husten.

Thermale Reaktion. Bei allen Kühen war bei den Endotininjektionen ein leichtes Ansteigen der Temperatur auf $C$ 1,01) und C $2,0^{2}$ ) zu beobachten. Auf $\mathrm{C}_{\left.4,0^{3}\right)}$ dagegen eine Depression in gleicher Stärke, resp. stärker. Auf D $0,5^{4}$ ) war das Ansteigen der Temperatur geringer, die Depression jedoch stärker. Auf D 1,05), D 2,06), D 4,07), D 8, $0^{8}$ ), D 10,09) war keine thermale Wirkung mehr zu konstatieren. Im allgemeinen kann gesagt werden, dass die Kurve auf Dosen bis D 1,0 sich unter der normalen Linie hielt; über D 1,0 dagegen auf derselben. Diese ausgesprochenen und charakteristischen Schwankungen der Temperaturkurve waren bei einer Kuh (Ada), die auf Bovotuberkulin Behring (Enddosis) stark reagierte, im Gegensatz zu den anderen nicht zu bemerken.

Allgemeinbefinden während der Behandlung. Der Appetit war bei sämtlichen Kühen gut. Das Quantum der gemolkenen Milch nicht herabgesetzt. Die Kühe waren zusehends voller geworden als vor der. Endotinbehandlung. Die matte Haarfarbe war einer glänzenden gewichen. Von den 8 kranken Kühen husteten 4 . In den ersten Tagen des Monat März hatten 2 aufgehört zu husten, später auch die übrigen. (Bericht des Farmers.)

Letzte Kontrollimpfung. 40 Tage nach der letzten Endotininjektion wurden die Kühe wiederum mit Be h r in g schem Bovotuberkulin geimpft, diesmal mit der 4 fachen Dosis, d. h. mit $2,0 \mathrm{~g}$. 7 Kühe reagierten nicht mehr.

Sektionsprotokoll. (Dr. Hinderson. Kopenhagen und Helsingsfors, Veterinär-Institut.) Lymphoglandulae mediastinalis et bronchiales. Teile der Lunge.

Makroskopiseh. Die Lymphdrüsen etwas vergrössert; auf dem Durchschnitt grössere und kleinere verkäste Herde enthaltand, welche teilweise mit einer derben fibrösen Kapsel umgeben sind. Kalksalze kaum vorhanden. Lunge normal bis auf einige atelektatische Partien, ohne spezifisch pathologische Veränderungen.

Mikrosk opisch. Die Kapseln sind rein bindegewebig; keine nennenswerte Zellproliferation. Spärliche Tuberkelbazillen sind im Ausstriche nachzuweisen.

1) $10 \mathrm{mg}$. 2) $20 \mathrm{mg}$. 3) $40 \mathrm{mg}$. 4) $50 \mathrm{mg}$. 5) $100 \mathrm{mg}$. 6) $200 \mathrm{mg}$. 7) $400 \mathrm{mg}$. 8) $800 \mathrm{mg}$. 9) $1000 \mathrm{mg}$. 
In einem anderen Falle war das Bindegewebe besser entwickelt und die Einlagerung der Kalksalze reichlicher. Der Tierversuch fiel vollständig negativ aus.

Somit hatte der Tierversuch ergeben, dass das Endotin ganz ausserordentlich günstige Resultate bewirkt, nicht nur im Sinne einer klinischen, sondern auch anatomischen Heilung. Dabei war auch die Widerstandskraft der Tiere gegenüber hohen Dosen von Tuberkulin zu konstatieren. (Bovotuberkulin Behring 2,0.)

Hierbei sei noch bemerkt, dass die von den behandelten Tieren stammenden Kälber bei der Sektion sich als gesund herausgestellt hatten.

Wird dem gegenüber in Betracht gezogen, dass das Endotin bəi der 'Aktivitätsprüfung, selbst bei $100 \mathrm{mg}$, das tuberkulöse Meerschweinchen nicht zu töten vermag, so muss anerkannt werden, dass das Endotin keine toxischen Eigenschaften entwickelt, wio die anderen Tuberkuline; dass es also unschädlich ist. sowohl für Mensch als Tier.

Als weitere Bekräftigung der Wirksamkeit des Endotins mögən die Untersuchungen über den Opsoninindex dienen.

Es konnte nachgewiesen werden, dass der Opsoninindəx rom zweiten Tage ab, nach stattgehabter Endotininjektion, zu steigən beginnt, wobei die Kranken, sowohl subjektiv als objektiv, sich erheblich besserten.

Die ganz exakt feststehenden Tatsachen, sowohl der klinischen Beobachtung, als auch des Tierexperimentes, hönnen nicht mehr zwei Deutungen zulassen; die spezifische Substanz oder das heilende Agens besitzen wir im Endotin.

Der Zwiespalt, der bis jetzt in sämtlichen Arbeiten wahrgənommen wird, wo es sich um klinische Beobachtung der Tuberkulinwirkung und der entsprechenden Erklärung handelt, darf ohne weiteres dem Umstand zugeschrieben werden, dass man es mit keinem einheitlichen Präparat zu tun hatte, sondern mit einem Mittel, dessen Komponenten, jedes in seiner Art, den Organismus verschieden beeinflussen mussten.

Wie auch immer die Methodik der Herstellungsweise der verschiedenen Tuberkuline sein mochte, das heilende Prinzip war überall das Endotin. Unzweckmässig und schädlich wurde ein Tuberkulin, sobald es sich um Kranke handelte, die schon stark mitgenommen waren, ganz unabhängig von der Ausbreitung des anatomischen Prozesses. Hier musste sich natürlich jenes Bild wiəderholen, das wir bei der Aktivitätsprüfung beim Tier sehen: di e Reakti o n konnte das höchste Extrem der Erschöpfung er- 
reichen. Die Praxis half sich einfach; sie griff zu der Minimaldosierung. Und so möchte man beinahe glauben, der Heilzwick steh in zweiter Linie - die Reaktion zu vermeiden um jeden Preis - - in erster Linie. Die Gegner einer Tuberkulinbehandlung hatten gewissermassen recht. Der Gewinn schion zu gering. Viele Tuberkulinfreunde begnügten sich mit dem Ausbleiben der Reaktion. Schliesslich wurde noch die Immunitätslehre herangezogen, um einє theoretische Begründung zu haben; in dizser Hinsicht fehlen jedoch die Beweise. Ein ideales Tubarkulin ist ein solches, das nicht schadet. Das ist aber nicht gleichbedeutend mit der Methode der kleinsten Dosen. Es ist fraglos, dass das stärkste Gift in entsprechender Verdünnung keine schädigenden Eigenschaften mehr entwickelt; es wird indifferent.

Wenn nun von verschiedenen Seiten geltend gemacht wird, dass für die Tuberkulintherapie, bis zu gewissen Dosen, keinerlei Bedürfnis vorliege für albumosen-, pepton- und glyzerinfreien Präparaten, so würde man sich damit nur in dem Falle für einverstanden erklären können, wenn bewiesen wäre, dass die Nebenprodukte in einem Tuberkulin nicht nur micht schädlich wirken, sondern im Gegenteil nutzbringend seien. Nun steht es aber fest, dass Albu. mosen und Pepton, sowohl beim Gesunden, wie beim Tubrrkulösen, Fieber erzeugen können; dass der Tuberkulöse selbst auf kleinere Doser: Reaktion zeige als der Gesunde. Dass Tuberkulinwirkung nicht Peptonwirkung ist, versteht sich von selbst. Das Tuberkulin enthält eben neben dem Pepton noch die wirksame Substanz. Deswegen wird auch beim Tuberkulin die Reaktion viel rascher eintreten, resp. auf kleinere Dosen.

Der Endzweck einer jeden Therapie ist die Heilung, und nur die exakte klinische Beobachtung kann hier Klärung bringen.

Die Beurteilung des Wertes eines Heilmittels wird in erster Linie abhängig sein von seiner effektiven Wirksamkeit auf den Krankheitsprozess und in zweiter Linie von den Nebənerscheinungen, die sich dabei geltend machen. Sind letztere stärker als der Heileffekt, so wird es geschehen können, dass der Organismus zu stark geschädigt wird, weshalb von der Benutzung des Mittels Abstand genommen werden muss. Wir müssen aber daran festhalten, dass nur indifferente Körper den Organismus gar nicht beeinflussen, sonst aber immer eine Wirkung entsteht, die eine Umgestaltung des gegebenen Zustandes involviert. Neben der spezifischen, heilenden Aktion wird demnach auch eine unerwünschte Änderung in den Organfunktionen $z u$ beobachten sein. Wie weit nun letzteres als Schädigung zu bezeichnen ist, lässt sich nicht 
ohne weiteres, vom theoretischen Standpunkt, sagen; es gehören dazu Ergebnisse einer objektiven Beobachtung.

En d o t in und Allgemeinzustand: Es liegen zahlreiche Berichte vor, dass die Kranken weder während, noch nach der Endotinbəhandlung bettlägerig werden. Es scheint mir das von grosser Bədeutung zu sein. Selbst die sehr schweren Fälle der Tuberkulose zeigten kein Erscheinungen, die auf gestörte Funktionstätigkeit irgendwelcher Organe hindeuteten. Die meisten Patienten waren aber in ihrer üblichen Tätigkeit nicht behindert und konnten ihre tägliche Arbeit verrichten. Selbst von den Kranken des III. Stadiums wird angegeben, dass sie in ihrem Allgemeinbefinden keinerlei Störungen erlitten (Gaikowitsch, Koch).

Dies wären aber nur Beweise einer unschädlichen Wirkung. Dass aber noch eine äusserst günstige Wirkung erzielt wird, dafür sprechen. die Untersuchungen vieler Autoren (P e r o t t). So konnte nachgewiesen werden, dass parallel mit der Endotinbehandlung der Opsoninindex ansteigt, und dass bei den Kranken eine bedeutende Besserung des Allgemeinbefindens eintrat, sowohl subjektiv, als auch objektiv (Michailowskaja).

Schon auf kleine Dosen war diese Besserung zu verzeichnen; zuweileı waren sie überraschend scharf ausgesprochen ( $\mathrm{C} \mathrm{h} \mathrm{r} \mathrm{eibn} \mathrm{e} \mathrm{r).}$ Ohne Unterschied der Krankheitsform konnte eine solche günstige Beeinflussung in $80-90 \%$ der behandelten Patienten konstatiert werden (Gabrilowits ch, Fed or of f).

Aus diesen Untersuchungen war schon ersichtlich, dass das Endotin eine spezifische Substanz darstellt, mit der Heileffekte erzielt werden, wie sie die anderen Tuberkuline nicht geben; dass wir es gewissermassen mit einem Gegengift der Tuberkulose zu tun haben (Neumann, Deal).

End ot in und Körpergewicht. Sämtliche Arbeiten, die von den Erfolgen eines Heilmittels gegen Tuberkulose sprechen, behandeln die Körpergewichtszunahmen in besonders ausführlicher Weise. Und doch kommt letzteren oft nur relative Bedeutung zu. Sehr viel hängt natürlich davon $a b$, in welchen Verhältnissen der Kranke vor der Kur gelebt und wie dieselben während der Kur waren. Ebenso wichtig sind natürlich die Angaben über sein Körpergewicht vor der Behandlung, über die Krankheitsform, über Fieber, Komplikationen etc.

Ich habe bei der Endotinbehandlung Zunahmen in $96 \%$ aller Fälle erzielt. Das ist an und für sich natürlich nicht massgebend, um von einem grossen Heilwert zu reden. Eine massgebende Begründung findet das Mittel aber darin, dass von den mit Endotin behan- 
delten Kranken nur etwa $10 \%$ zu den leichteren Fällen gehören, und dass die meisten vor dem Beginn der Kur bereits längere Zeit hyigenisch-diätetisch behandelt wurden, während welcher sie schon an Körpergewicht zugenommen hatten. Weiter ist beachtenswert, dass bei den meisten, Komplikationen von seiten des Digestionsapparates, des Nervensystems bestanden; viele litten ausserdem an Kehlkopftuberkulose; bei den meisten aber war die Körperwärme gesteigert.

Die äusseren Verhältnisse waren zudem vor der Kur nicht schlecht, da die meisten Kranken dem besseren Mittelstand angehörten. In den Berichten aus Nord und Süd des weiten Reiches finden wir annähernd dieselben Zahlen. Wir finden Zahlen von 100\% Körpergewichtszunahmen unter Soldaten ( $\mathrm{Z}$ lat tow e $\mathrm{r} \mathrm{ch}$ ow nikoff), 90\% unter Kranken der Krim (Fedoroff), bis zu 15 Pfund im Mittel unter der ärmeren Bevölkerung Moskaus (Lap schin). In der ambulatorischen Behandlung bei durchweg armen Handwerkern Petersburgs wurden noch 850,0 Körpergewichtszunahmen erzielt (Gaikowitsch).

Bei verzweifelten Fällen von unheilbarer Tuberkulose unter der Bevölkerungs New Yorks und unter den denkbar ungünstigsten Verhältnissen wurden noch Körpergewichtszunahmen notiert (D e a l). Dazu kommt noch, dass das Ansteigen des Gewichts schon auf kleine Endotindosen zu beobachten war ( $\mathrm{Neumann}$ ). Zuweilen wurden ungewöhnlich grosse Zunahmen konstatiert, bis zu 20-30 Pfund ( $\mathrm{K} \circ \mathrm{ch}$ ).

Puls und Respiration bei der Endotin behandlung. Zu den am meisten unerwünschten Erscheinungen der ersten Tuberkulinära, welche bei der Alttuberkulinbehandlung in den Vordergrund traten, gehören beschleunigter Puls und gesteigerte Respirationsfrequenz.

Mit dem Heruntergehen in der Dosierung wurden diese Symptome seltener, obgleich sie immer noch auftreten. Nun ist aber beschleunigter Puls und gesteigerte Respirationsfrequenz schon an und für sich eine Begleiterscheinung der Tuberkulose und sollen daher Eingriffe, die sie noch steigern, durchaus vermieden werden.

Bei der Endotinbehandlung tritt dieses Symptom nie auf. Im Gegenteil, es treten Besserungen auf, die dadurch gekennzeichnet sind, dass der Puls langsamer wird und die Respiration seltener und tiefer.

In bezug auf den Puls wird angegeben, dass der Puls bei dieser Behandlung in $71 \%$ aller behandelten Fäle ruhiger wurde (Fedo- 
roff). Dass der frequente Puls zur Norm zurückkehrte, wird als eine auffallende Erscheinung angegeben (Gordon).

Endotin und Appetit. Obgleich man niemals den subjektiven Symptomen allzu grosse Beachtung schenken sollte, so scheint es doch, dass das Endotin auch den Appetit stark anregt.

Schon nach ganz kleinen Dosen war mir das aufgefallen, obgleich die Kranken ihre gewohnte Lebensweise in keiner Weise geänderi hatten. Stets war ihm eine grössere Gewichtszunahme gefolgt. Auch von anderer Seite swird das bestätigt (Perott). Es konnte sogar ziffermässig festgestellt jwerden, dass etwa $50 \%$ der mit Endotin behandelten Kranken eine Besserung des Appetits zeigten (Fed or off).

End otin und Schweiss. In naher Beziehung zum Fieber steht der Schweiss. Es muss jedoch hervorgehoben werden, dass die Nachtschweisse nicht immer in direkter LAbhängigkeit vom Fieber stehen, da sie auch dann vorkommen, wenn die Temperatur bereits normal geworden. Die Ursache für ihr Auftreten ist vielmehr in der Intoxikation des Organismus mit Zerfallsprodukten zu suchen.

Je schwerer der Krankheitsprozess, um so häufiger und hartnäckiger die Nachtschweisse. Bei der Endotinbehandlung schwinden die Nachtschweisse, ganz einerlei ob im II. oder im III. Stadium, sehr rasch.

Ein Schwinden der Nachtschweisse wurde von anderer. Seite bei der Anstalts-, Hospital- und ambulatorischen Behandlung konstatiert (Zlat towerchownik of f, Deal).

End ot in und Husten. Husten ist bei den meisten Tuberkulösen vorhanden; nach meinen Beobachtungen in etwa 97-98\%. Des Nachts sistiert der Husten meist, um am Morgen und Abend sich besonders stark geltend zu machen. Am Tage oder in der Nacht werden diejenigen Kranken von Husten gequält, bei denen er trocken ist oder nur spärlichen Auswurf gibt. Der trockene Husten ist səhr schwer zu behandeln, namentlich dann, wenn der Ruiz in den oberen Luftwegen sitzt. Heftiger Husten mit profuser Schleimabsonderung wird meist im III. Stadium der Erkrankung beobachtet, ungefähr in $62 \%$ der Fälle, aber auch im II. Stadium ist er noch häufig (37\%), während er im I. Stadium fast gar nicht vorkommt. Husten von schwacher Intensität ist im II. Stadium am häufigsten (44\%), dann im I. Stadium (25\%) und etwas seltener im III. Stadium (23\%), wogegen der mittelstarke Husten bei Kranken des I. Stadiums weit häufiger vorkommt $(38 \%)$ als bei solchen des II. Stadiums (19\%) oder des III. Stadiums (16\%). 
Der Husten ist als ein Hilfsakt der Natur anzusehen, der den Zweck hat, die Entzündungs- und Zerfallsprodukte aus der Lunge zu entfernen. Je nachdem, ob der Entzündungsherd zentral gelegen ist oder in Verbindung steht mit einem oder mehreren Bronchien, wird er trocken oder schleimig sein. Lokale Reize im Kehlkopf veranlassen meist Hustenanfälle, die eine energische Behandlung notwendig machen. Der trockene Husten ist aber nicht nur unangonehm für den Kranken, sondern direkt schädlich, indem er häufig zu Brechbewegungen und Erbrechen während oder nach der Mahlzeit führt. Er führt damit nicht nur zu subjektivem Unbehagen, sondern die Ernährung des Kranken leidet darunter. Dio Furcht vor dem Erbrechen bewirkt eine geringere Nahrungsaufnahme und eine Schwächung des allgemeinen Ernährungszustandes.

Bei der hygienisch-diätetischen Behandlung gelingt es nach meinen Beobachtungen, den Husten günstig zu beeinflussen. Es vergehen zwar oftmals mehrere Monate, eho das erreicht wird, aber die verschiedenen Behandlungsmethoden führen doch meist zum Ziel. Bei Kranken des I. Stadiums wurde der. Husten geringer in 25\% ; er schwand vollständig in $38^{\circ}, 0$. Im II. Stadium wurde er schwächer während der Kur in $50 \%$ und hörte auf in $130 \%$. Im III. Stadium war der Husten während der Kur kein einziges Mal geschwunden, wurde aber geringer in $31 \%$.

Bei der End o ti n behandlung konnte bei 45\% ein gänzli ches Schwinden des $\mathrm{H}$ ustens konstatiert werden; bei $50 \%$ wurde er geringer (Fed or off, Deal).

Der Auswurf, der in naher Beziehung zum Husten steht, wird durch die hygienisch-diätetischen Massnahmen ebenfalls günstig beeinflusst. Im II. Stadium hörte er ganz auf in $130 \%$; selbst im III. Stadium verschwand er in $4 \%$.

Bei der Endotin behandlung schwand er ganz in $15-20 \%$ und wurde bei den meisten Kranken geringer (Fed o r off, Dea l, Gabrilowitsch). Husten und Auswurf schwanden bei Kranken des I. und II. Stadiums in etwa $50 \%$ ( $\mathrm{K}$ och).

Endotin und Lungenblutungen. Die Ursache für das Auftreten der Blutungen liegt im tuberkulösen Prozess selbst, der zur Zerstörung des Lungengewebes führt. Bei seiner Entwickelung und beim Weiterschreiten können natürlich Blutgefässo angebohrt, und so Blutungen bewirkt werden. Ist die Gefässwand einmal krankhaft verändert, so können selbst äussere Momente eine Blutung veranlassen. Heftige Erschütterung des Brustkorbes durch Stoss oder Fall, auch starker Husten, Muskelanstrengungen, atmosphärische Luftdruckschwankungen etc. Zieht man somit dies alles in Betracht, 
so muss es zum mindesten ungerecht erscheinen, wenn man, wie das so häufig behauptet wird, dem Tuberkulin alle Schuld für das Eintreten einer Blutung zuschreibt. Und so hat es beinahe den Anschein, als ob mit dem Auftreten einer Blutung nach stattgehabter Injektion die Autoren alle Überlegung verloren hätten. Denn aus der blossen Tatsache der Erscheinung folgt ja noch gar nicht, dass das Tuberkulin die veranlassende Ursache war.

Bei der Endotinbehandlung sind Blutungen äusserst selten. Damit soll nicht gesagt sein, dass sie überhaupt nicht auftreten könn’n. Andererseits sind aber Fälle bekannt, wo Kranke, die für Lungenblutungen besonders disponiert waren, diese nach der Endotinbehandlung ganz verloren. Trotz der ausgesprochenen Herdreaktion wurden Lungenblutungen weder in der Anstalt-, noch in der Hospital- oder ambulatorischen Praxis beobachtet.

Endotin und Tuberkelbazillen. Die mikroskopische Untersuchung des Auswurfs ergibt ein stets wechselndes Bild bei ein und demselben Kranken in bezug auf die Zahl der Tuberkelbazillen. Es kann deshalb die Abnahme der Tuberkelbazillen nur bedingten Anspruch auf Beachtung verdienen, wenn diese Abnahme keine dauernde ist. Als sehr günstiges Zeichen ist hingagen das gänzliche Schwinden der Tuberkelbazillen aus dem Auswurf zu bezeichnen.

Meine Beobachtungen in dieser Beziohung ergaben für das Endotin : 55-60\% gänzliches Schwinden der Tuberkelbazillen und 35\% dauernde Abnahme. Andere Autoren geben an: 50\%, resp. 42\% (Gaikowitsch). Wieder andere $12 \%$, resp. 24\% (Zlatowerchownikoff). Selbst bei der ambulatorischen Behandlung sind noch $25 \%$, resp. $25 \%$ beobachtet worden (G a ik ow it s ch).

Sehr güstige Resultate waren auch bei Schwerkranken zu notierer. ( $D$ e a l).

End ot in und katarrhalische Erscheinungen. Die Heilwirkung des Endotins äussert sich am prägnantesten im Schwinden der katarrhalischen Erscheinungen in den Lungen. Zuerst entsteht eine Exazerbation im entzündeten Gewebe, darauf eine Verringerung der katarrhalischen Erscheinungen, und schliesslich gänzliches Schwinden derselben. Diese konstanten Ergebnisse einer langjährigen Beobachtung kehren überall wieder; bei vielen Kranken rascher, bei anderen langsamer.

Gänzliches Schwinden der katarrhalischen Erscheinungen konnte ich konstatieren in $60 \%$, eine Verringer ung derselben in $35 \%$. Zusammen in $95 \%$ eine sichtbare, unzweifelhafte, günstigø Beeinflussung des lokalen Prozesses. Ein gänzliches Schwinden wurde auch noch häufiger notiert und zwar in $71 \%$ ( $\mathrm{B} \ddot{o} \mathrm{hm}$ ), aber 
auch seltener, 50\% (Gaikowits ch). Ambulatorisch konnte das erreicht werden in $44 \%$, eine Verringerung in 32\%, zusammen also in $76 \%$ (Gaikowits ch). Eine grosse Besserung, sowohl quantitativ, als auch qualitativ, zeigten die meisten mit Endotin behandelten Kranken (Fed or of f).

In der Hospitalpraxis war eine solche Besserung in $66 \% \mathrm{zu}$ verzeichnen (Lapschin).

Sämtliche Autoren, die mit dem Endotin gearbeitet haben, sind in dieser Hinsicht einer Meinung, dass es die katarrhalischen Erschein ungen zum Schwinden bringt oderstarkvermindert (Neumann, Deal petc.). Alle Kranken des II. und III. Stadiums hatten eine Besserung aufzuweisen $(\mathrm{K} \circ \mathrm{c} \mathrm{h})$.

Endotin und Infiltrate. Bei weitem geringer ist die Wirkung des Endotins auf bereits längere Zeit bostehende Infiltrate. Dass aber auch hier eine teilweise Aufsaugung oder Verringerung des infiltrierten Gewebes erreicht wird, unterliegt keinem Zweifel (B ö h m).

Selbst in der ambulatorischen Praxis konnte eine solche Wirkung nachgewiesen werden und zwar in 50\% der behandelten Fälle (Gaikowits ch).

Selbst bei Schwerkranken war diese Tatsache noch nachweisbar (De a l).

End otin und Fieber. Man kann mit Recht sagen, dass die Tuberkulose fast nie ohne Fieber verläuft. Ein jeder Tuberkulöse wird einmal erhöhte Temperaturen gehabt lhaben. Natürlich sind die Temperatursteigerungen abhängig von dem Erkrankungsprozesse und verschwinden zeitweise oder ganz, sobald der Kranke der Besserung oder Heilung entgegengeht.

Das Auftreten von Fieber bei Tuberkulösen, auch in den beiden ersten Stadien der Erkrankung, ist stets mit einer Sistierung der Sputumabsonderung verbunden. Sobald letztere wieder reichlicher wird, sinkt auch das Fieber. Dieser Vorgang wiederholt sich so oft, dass eine jede Krankengeschichte besonders angeführt werden könnte, um dieses zu bestätigen.

Freilich wird auch Fiebersteigerung bei reichlichem Auswurf beobachtet, aber dann handelt es sich meistens um schwere Fälle des III. Stadiums, wo zahlreiche Herde in der Lunge nachgewiesen werden können.

Das Prinzip der Fieberbehandlung ist der chirurgischen G:schwürsbehandlung vergleichbar. Wie bei dieser muss dafür gesorgt werden, dass die Zerfallsprodukte und Eitermassen rasch entfernt werden. Als beste Behandlungsmethode ist die Endotinanwendung zu nennen. 
In dieser Hinsicht kann vom Endotin gesagt werden, dass eine Entfieberung stets da eintritt, wo es sich nicht um akut verlaufende Prozesse handelt (B ö h m).

Bei Temperaturen, die $38,5^{\circ}$ nicht überstiegen, konnte eine Entfieberung in $50 \%$ erzielt werden ( $\mathrm{Z}$ lat ow erchownik of f). In der ambulatorischen Praxis war das in 66\% zu erreichen (Gaikowits ch); temperaturherabsetzend in $30 \%$ (bis zur Norm). Jedenfalls wird die Temperatur normal oder sie wird geringer bei den meisten Kranken ( $\mathrm{F}$ ed o r of f).

Eine solche Wirkung konnte schon nach den ersten Dosen nachgewiesen werden ( $N$ eumann, Gordon, Deal etc.).

\section{Endotin bei den verschiedenen Formen der Lungentuberkulose.}

Tuberculosis pulm. sicca. Pathologisch-anatomisch ist diese Form charakterisiert durch Bildung fibröser Knötchen, die zum Teil verkäst sind. Klinisch ist eine Dämpfung über der Klavikula und Spina zu notieren. Die Atmung ist verschärft, rauh, oder es besteht verlängertes Ekspirium. Kein Rasseln. Zuweilen Krepitieren. Keine Komplikationen spezifischer Natur. Heilung: Vernarbung. Klinisch: Dämpfung geringer, Atmung mehr vesikulär. (Kein Krrepitieren.) Leistungsfähigkeit nach der Kur durehweg erhalten.

Die Endotin behandlung ergab nach meinen Beobachtungen bei dieser Form der Krankheit etwa 80\% Heilung und 20\% Besserung. Andere Autoren kommen zu demselben Resultat (Bö h m). Einige geben grössere Zahlen an (Hirschberg, 100\% Heilung).

Tuberculosis pulm. catarrhalis. Pathologisch-anatomisch wird diese Form charakterisiert durch den Übergang der fibrösen Knötchen in käsige Massen und Bildung exsudativ-käsiger Herde. Klinisch: Dämpfung über dem Oberlappen, den Angulus scap. nicht überschreitend. Atmung abgeschwächt oder Exspirium. Krepitieren. Komplikationen in 15\%. Heilung: Neugebildetes fibröses Gewebe zwischen krankem und gesundem Gewebe eingelagert. Klinisch: Kein Krepitieren, Dämpfung geringer. Leistungsfähigkeit meist voll erhalten.

Die Endotin behandlung ergab: Heilung in 30\%, Besserung in 70\%. Ähnliche Angaben werden von anderen Autoren gemacht (Rostoschinsky, Böhm, Hirschberg, Lapschin).

Tuberculosis pulm. fibrosa. Pathologisch-anatomisch: weiteres Vordringen des Käses, Verflüssigung desselben, Durchbrechung der Kapsel und Bildung des tuberkulösen Geschwürs. Klinisch : Dämpfung des Oberlappens, zum Teil des Unterlappens, Angulus nicht über- 
schreitend. Krepitieren oder kleinblasiges Rasseln. Komplikationen in 25\%. Heilung: Käsige Massen entfernt, Reinigung des Geschwürsgrundes, Häutchenbildung, Schrumpfung und Verkleinerung der Höhle. Klinisch : Kein Rasseln, Dämpfung geringer, Atmung bronchovesikulär. Leistungsfähigkeit in $70 \%$ erhalten.

Bei der Endotin behandlung wurde erzielt: Heilung in 35\% ; Besserung ebensoviel.

Tuberculosis pulm. ulcerosa. Pathologisch-anatomisch: Verkäsung und Zerfall schreiten in der Höhle weiter fort, ergreifen benachbartes Gewebe und bilden grössere Höhlen. Klinisch: Dämpfung über dem Oberlappen, zum Teil Unterlappen. Tympanitisch. Atmung: amphorisch. Grossblasiges oder metallischas Rasseln. Komplikationen in 40\%. Heilung: Die Höhle reinigt sich, die Wände schrumpfen. Kein Rasseln, Atmung mehr bronchial. Ieistungsfähigkeit erhalten in $35 \%$.

Bei der Endotin behandlung werden noch bei dieser Form Heilungen erzielt und zwar in 25\%. Dieselben Zahlen werden auch von anderen Autoren angegeben ( $\mathrm{B}$ ö h m). Besserung ist in 30\%0 zu erreichen: Gabrilowitsch, Böhm, Zlatowerchowni koff $(33 \%)$, Lapschin $(35 \%)$. Viel grössere Ziffern sind anderwärts notiert: Hirschberg $(700 \%)$, Deal etc.

\section{Endotin bei Kehlkopituberkulose.}

Die Kehlkopftuberkulose muss als eine recht schwere Komplikation der Lungenphthise betrachtet werden, da es a priori nicht leicht zu sagen ist, welchen Verlauf und Ausgang dieses Leiden nehmen wird. Sehr oft erfahren die Kranken von ihrem Kehlkopfleiden erst nach der laryngoskopischen Untersuchung, da ihr subjektives Befinden von dieser Seite in keiner Weise gestört war. Die Erfahrung lehrt nun, dass die Heilbarkeit der Larynxtuberkulose in der Mehrzahl der Fälle in direktem Verhältnis steht zur Ausbreitung des Lungenprozesses. Je schwerer die Lungenerkrankung, um so ungünstiger die Prognose für die Kehlkopftuberkulose und umgekehrt.

Die Endotin behandlung ergab fast durchweg günstige Resultate, selbst in Fällen stärkerer Ausbreitung des Lungenprozesses (Lebedeff). Ohne irgendwelche lokale Behandlung schwanden die Kehlkopfaffektionen, die Infiltrate auf den Stimmbändern etc. (B l u menau). Ẹs wurden einzelne Fälle einer ganz ausgezeichneten Heilung beobachtet (Rostos chinsk y). Im allgemeinen wurden überall Besserungen, Schwinden der Infiltrate und der Hyperämien konstatiert. 
So wird berichtet, dass die Stimme nach der Behandlung mit dem Endotin wieder rein und volltönend wurde.

Endotin und Nierentuberkulose. Bekanntlich ist die Prognose der Darm- und Nierentuberkulose gleich schlecht. Eine wirkliche Heilung ist fast ganz ausgeschlossen.

Die Endotinbehandlung scheint aher auch hier Bemerkenswertes zu leisten. So wird berichtet ( $\mathrm{raikow}$ its $\mathrm{ch}$ ), dass bei der Niarentuberkulose die Schmerzen beim Urinieren, die früher sehr heftig waren, während der Endotinhehandlung ganz aufhörten. Die Tuberkelbazillen verschwanden aus dem Harn, das Eiweissquantum verringerte sich bedeutend. Schliesslich konnte sogar Körpergewichtszunahme verzeichnet werden.

Endotin bei Knochen-und Gelenktuberkulose. Die vorliegenden Berichte in bezug auf diese Formen der Tuberkulose sind sehr günstig. Es wurden in der Hospitalpraxis unter den allerungünstigsten Bedingungen sehr schöne Erfolge erzielt (B lumenau). Das gleiche war zu konstatieren bei sehr schweren Fällen (D e a l).

Endotin und Lymphar üsentuberkulose. Die Lymphdrüsentuberkulose ist eine sehr häufige Erscheinung, ganz besonders häufig im jugendlichen Alter; aber auch bei Erwachsenen tritt sie nicht selten auf.

Die Endotinbehandlung gibt hier ganz besonders schöne Erfolg:s. So konnte ein gänzliches Schwinden der grossen Lymphdrüsenpakete konstatiert werden. Während für solche ausgebreitete Herde eine längero Behandlung erforderlich war, konnten kleinere Lymphdrüsen sehr rasch zum Schwinden gebracht werden.

Endotin inder ambulatorischen Behandlung. Es ist bezeichnend, dass kein einziges der vielen Tuberkuline, namentlich in der ambulatorischen Praxis, so leicht zu handhaben ist, wie das Endotin. Alle Autoren haben ganz vorzügliche Resultate erzielt (Blumenau, Gaikowitsch, Lapschin etc.). Es wird angegeben. dass es a usgezeichnetwirkt, sehr gleichmässig und dabei absolut unschädlich sei. Es ist deshalb leicht anweridbar bei der arbeitenden Klasso der Bevölkerung (Gor don). Da das Endotin absolut unschädlich ist, so erscheint es mir als das beste Mittel für die ambulatorische Praxis; jegliche Temperaturmessung kann hier weggelassen werden. Es fallen dabei weg nicht nur Tuberkulinschädigungen, sondern auch Schädigungen ökonomischer Natur.

Endotin in der Hospitalbehandlung. Selbst in ungünstig gestellten Hospitälern war die Endotinwirkung ganz ausge. 
zeichnet (Blumenau, Dea l, Lapschin etc.). Das scheint mir ein guter Beweis für die Wirksamkeit des Präparates zu sein. Hier fallen natürlich alle jene Momente weg, die bei seiner Anwendung neben der Anstaltsbehandlung geltend gemacht werden. Es werden da stets Bedenken entstehen, was auf Rechnung des Endotins, und was auf Rechnung der hygienisch-diätetischen Kur zu schreiben ist. Namentlich müssen solche Zweifel entstehen, wenn der Erfolg der Behandlung eine Besserung des ,Allgemeinzustandes ergibt, ohne dass der lokale Prozess in eklatanter Weise sich geändert hätte. Bei der Hospitalbehandlung, bei der bekanntermassen, namentlich da, wo die hygienischen Verhältnisse schlecht sind, etwa $100 \%$ Mortalität $\mathrm{zu}$ verzeichnen ist, fällt natürlich ein jedes Argument in diesem Sinne weg. Was da erreicht wird, ist ausschliessliches Verdienst des Mittels.

Endotin und Klima. Die Behandlung mit dem Endotin ist ganz unabhängig vom Klima, wie das übrigens auch gar nicht anders sein kann. Es soll aber nur darauf hingewiesen werden, dass die Luft, die die Kranken beständig einatmen, mancherlei Veränderungen erleidet, die von grosser Bedeutung ist für den Lungenprozess selbst. Ich meine die meteorologischen Schwankungen, die im Norden grosse Wellenbewegungen machen. Der Winter zeichnet sich nicht durch Beständigkeit aus; Frühling und Herbst sind im Norden sehr unbeständig und zeichnen sich durch feucht-kalte Witterung aus. Diess ist für die Kranken in keiner Weise günstig, da sie zu Katarrhen der Respirationswege Veranlassung gibt. Es muss deshalb als besonderes Verdienst der Endotinbehandlung bezeichnet werden, wenn die ambulatorische Kur unter solchen Verhältnissen erfolgreich durchgeführt werden konnte. Wir wissen ja, dass bei Erkältungen verschiedener Natur eine Tuberkulinkur meist unterbrochen werden muss. Wir müssen deshalb annehmen, dass die Endotinbehandlung trotz Erkältungen erfolgreich zu Ende geführt werden konnte. Die Angaben der Autoren stimmen auch darin überein (Blum en au).

\section{Indikationen und Kontraindikationen.}

Ich muss gestehen, dass ich keine Kontraindikationen kenne, bei welchen das Endotin nicht anzuwenden sei. Die Ansichten der verschiedenen Ärzte, die mit dem Mittel gearbeitet haben, lassen sich folgendermassen gruppieren: I. Kontraindikation bei Temperaturen über $38,0^{\circ}$. II. über $38,5^{\circ}$ (B l umenau, ChadjiI wa n off). III. Keine Kontraindikation ( $\mathrm{Per}$ ott). $\mathrm{Zu}$ der letzten Gruppe gehören z. B. Autoren, die das Endotin gerade in verzweifelten Fällèn anwenden (Neumann). Es wird auch 
angegeben, dass das Endotin indiziert sei bei hochfiebernden und schweren Fällen (Gordon). Zuletzt lesen wir, es sei indiziert bei schweren Fällen, bei welchen Tuberkulin bis jetzt überhauptkontraindiziert war (Deal).

Es scheint mir, dass einige Autoren die Tuberkulose unterschätzen. Wir müssen davon ausgehen, dass die Tuberkulose unter gewöhnlichen Verhältnissen eine $\mathrm{u} n \mathrm{~h}$ e il bare Krankheit ist. Warum sollen wir einige Kranke, die Hilfe vielleicht am meisten brauchen, ausschliessen und sie nicht mit Endotin behandeln? Es wäre das verständlich, wenn wir diese Fälle mit anderen uns zu Gebote stehenden Mitteln gegen die Tuberkulose heilen könnten, während das Endotin thermale Temperatursteigerung hervorrufen kann, die den Schwerkranken ungünstig beinflussen müsste. Wie verhält es sich aber damit in Wirklichkeit? Wir besitzen eben gar keine Mittel gegen solche Formen der Krankheit und sind deswegen darauf angewiesen, symptomatisch zu behandeln. Wo ist hier die Kontraindikation? Ganz im Gegenteil halte ich diese schwere Form der Krankheit geeignet für die Endotinbehandlung. Und zwar nicht deshalb, weil ich glaube, mit dem Endotin jede Form der Tuberkulose heilen zu können, sondern weil ich es für die Pflicht des Arztes halte, wenigstens einen Versuch zu machen, um den Kranken zu retten. Nun wäre das ja eine unnütze Arbeit, wenn es schon feststünde, dass das Endotin in solchen Fällen nichts leistet oder dass es schadet. Wir haben aber gesehen, dass viele Autoren gerade in $\mathrm{v}$ er $\mathrm{zw}$ e i felten Fällen zum Endotin ihre Zuflucht nehmen; as muss also doch irgend eine günstige Wirkung in diesen Fällen beobachtet worden sein. Ich kann aber auch selbst bestätigen, dass in einigen sehweren Fällen, bei Hochfiebernden, eine Entfieberung eintrat nach der Behandlung mit dem Endotin. Es sollte deshalb nie a priori von Kontraindikationen geredet werden; ein Versuch müsste immer gemacht werden.

\section{Endotinreaktion.}

Ich komme nun auf das vielbesprochene und heissumstrittene Thema der Reaktion zu sprechen. Es ist bereits oben erwähnt, dass die allgemeine Reaktion von den Ekdotinen herrührt und nur die thermale Reaktion, wenn sie überhaupt eintritt, als eine Folge der Endotinwirkung anzusehen ist.

Bei der Endotinbehandlung ist eine thermale Reaktion, also Elevation der Temperaturkurve, äusserst selten; und wenn sie eintritt, sehr gering (Schreibner). Selbst bei Injektionen von $200 \mathrm{mg}$ wurde sie nicht beobachtet (Chadji-I wan of f, Gordon, D e al). 
Unter 1549 Injektionen war eine Temperatursteigerung über $0,5^{0}$ nur in $2,5 \%$ zu registrieren.

Über $1,0^{0}$ wurde sie unter 2282 Injektionen nur in $1 \%$ beobachtet (Hirsch berg).

Nun steht es fest, dass bei der Endotinbehandlung neben der Temperaturelevation aucl eine Temperaturdepression zu beobachten ist, und zwar in etwa $25 \%$ der hehandelten Fälle. Diese temperaturherabsetzende Kraft des Fndotins ist sehr gross (Neumann) und tritt schon bei kleinen Dosen auf (Fedoroff). Was die Herdreaktion betriftt, so ist sie fast immer scharf ansgesprochen (Gordon, Rostoschinisy, Zlatowerchowni$k$ off). Ich behaupte, dass dort, wo sie besonders scharf ausgesprochen, der lokale Prozess sich rasch bessert. Es ist in solchen Fällenentschiedenfalsch, die Kurzunuterbrochen; es sei denn, die Lunge habe sich gereinigt. An der Einstich. stellewurden nie Infiltrate beobachtet (Koch), oder andere Entzündungsvorgänge, wie bei Alttuberkulin ( $\mathrm{F}$ edoroff).

Über Körpergewichtsrealitionen lässt sich überhaupt nur schwer reden, da dieselben, wenn sie wirklich vorkommen. sich unserer Kontrolle entziehen.

\section{Schlusssätze.}

1. Endotin ist staatlich geprüftes Alttuberkulin, auf chemischem Wege verändert. Laut chemischer Analyse besteht diese Veränderung in einer beträchtlichen Verminderung der stickstoffhaltigen Körper.

2. Beim Tierversuch ergah Endotin sehr günstige Resultate im Sinne einer klinischen und anatomischen Heilung. Bei der Aktivitätsprüfung entwickelt es jedoch keine toxischen Figenschaften, wie die anderen Tuberkuline.

3. Unter der Endotinbehandlung steigt der Opsoninindex.

4. Sämtliche Krankheitserscheinungen werden in günstigster Weise beeinflusst, nicht nur bei der Lungentuberkulose, sondern auch bei den anderen Formen.

5. Es hat sich gut bewährt bei der ambulanten Behandlung und bei der Verwendung in der Hospitalpraxis.

6. Es macht keine Allgemeinreaktion. 


\section{Literatur.}

I. Gabrilowitscb, Russky Wratsch. 1908. Nr. 37 .

B. Perott, Wratschebnaja Gazeta. 1908. Nr. 41.

Ges. Tuberkulin, Beiträge zur Kenntnis der Wirkungsweise des "Endotins". 1910. Heft 1.

E. Blumena u, Wratschebnaja Gazeta. 1909. Nr. 18 u. 19.

S. Chorol, Wratschebnaja Gazete. 1909. Nr. 18.

F. Böhm, Russky Wratsch. 1909. Nr. 34.

J. Sawolshskaja, Russky Wratsch. 1909. Nr. 34 .

M. Rostoschinsky, Russky Wratsch. 1909. Nr. 32.

R. Gaikowitsch, Russky Wratsch. 1909. Nr. 41, 46, 49 u. 51.

A. Michailow skaja, Russky Wratsch. 1909. Nr. 45.

I. Gabrilowitsch, Taberculosis. 1910. Nr. 11.

B. Zlatowerchownik off, Wojenny med. Journal. 1910. Nr. 2.

Hadji Ivan off, Terapewtitscheskoje Obozrenje. 1910. Nr. 3.

R. Gaikowitsch, Nowoje w Medizine. 1910. Nr. 4.

W. Hirsch berg, Peterb. med. Wochenschr. 1910. Nr. 9.

R. Gajkowjtsch, Russky Wratsch. 1910, Nr. 31 w. 32.

W. Ne uman n (aus der Klinı Prof. Neusser). Beiträge zur Klinik der Tuberkulose. Bd. 17. Nr. 1. 1910.

P. Fedoroff, Charkofsky med. Journal. Mai 1910.

R. Gaikowitsch, Wratschebnaja Gazeta. 1910. Nr. 38.

G ord on (Berlin), Deutsche med. Wochensehr. 1910. S. 1746.

Jahresbericht des Sanatoriums "Ambrock“, Deutschland, v. Schreibner.

Aus dem Rechenschaftsbericht des Sanatoriums "Halila" 1910.

A. I. Lapschin, Moskowsk, Obozr. 1910. Nr. 14.

P. G. Mesernizky, Wratsch. Gaz. 1901. Nr. 46 .

A. I. Lapschin, Wratsch. Gaz. 1910. Nr. 46.

Jochmann und Möllers, Zur Behandlung der Tuberkulose mit eiweissfreien

Tuberkulinpräparaten. Deutsche med. Wochenschr. 1910. Nr. 46.

Gabrilowitsch, Bemerkungen über das Endotin, die spezifische Substanz des

Ko chschen Alttuberkulins. Deutsche med. Wochenschr. 1911. Nr. 3.

Gordon, Deutsche med. Wochenschr. 1911. Nr. 3.

Wolff-Eisner, Berl. klin. Wochenschr. 1910. Nr. 48.

Gabrilowitscb, Berl. klin. Wochenschr. 1911. Nr. 5.

Koch, Vortrag in München. Münchn. med. Wochenschr.

Deal, Medical Record. 1910. Nr. 29.

Gabrilowitsch, Brïsseler Vortrag: Infektion, Erkrankang und Heilung. 1910.

Arbeiten aus dem Königlichen Institut für experimentelle Therapie zu Frankfurt

a. M. Herausgegeben von Dr. Ehrlich, II. Heft. R. Otto, Die staatliche

Prüfung der Heilsera. Jena 1906.

Behring, Beiträge zur experimentellen Therapie. Heft 5. S. VIII u. $\mathrm{XV}$.

L. Krehl u. M. Matthes, Deutsches Archiv für klinische Medizin. Bd. 54. 1895. Kühne, Zeitschrift für Biologie. 1893. Bd. I.

Matthes, zitiert nach A. Jolles, Chemie des Tuberkulins.

Hunter, The nature action and therapeutic value of the activ principles of

Tuberculin. Brit. Med. Journ. 25 July 1891. 

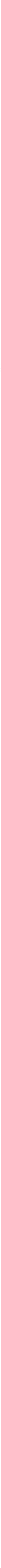

Brauer u. Lucius Spengler, Klinische Beobachtungen bei künstlichem Pneumothorax. 

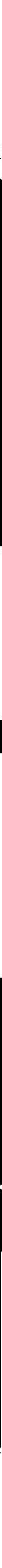

Brauer u. Lucius Spengler, Klinische Beobachtungen bei künstlichem Pneumothorax. 

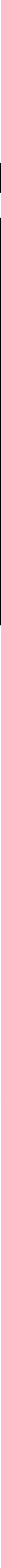

Brauer u. Lucius Spengler, Klinische Beobachtungeti tei künstlchem Pincumothorax. 

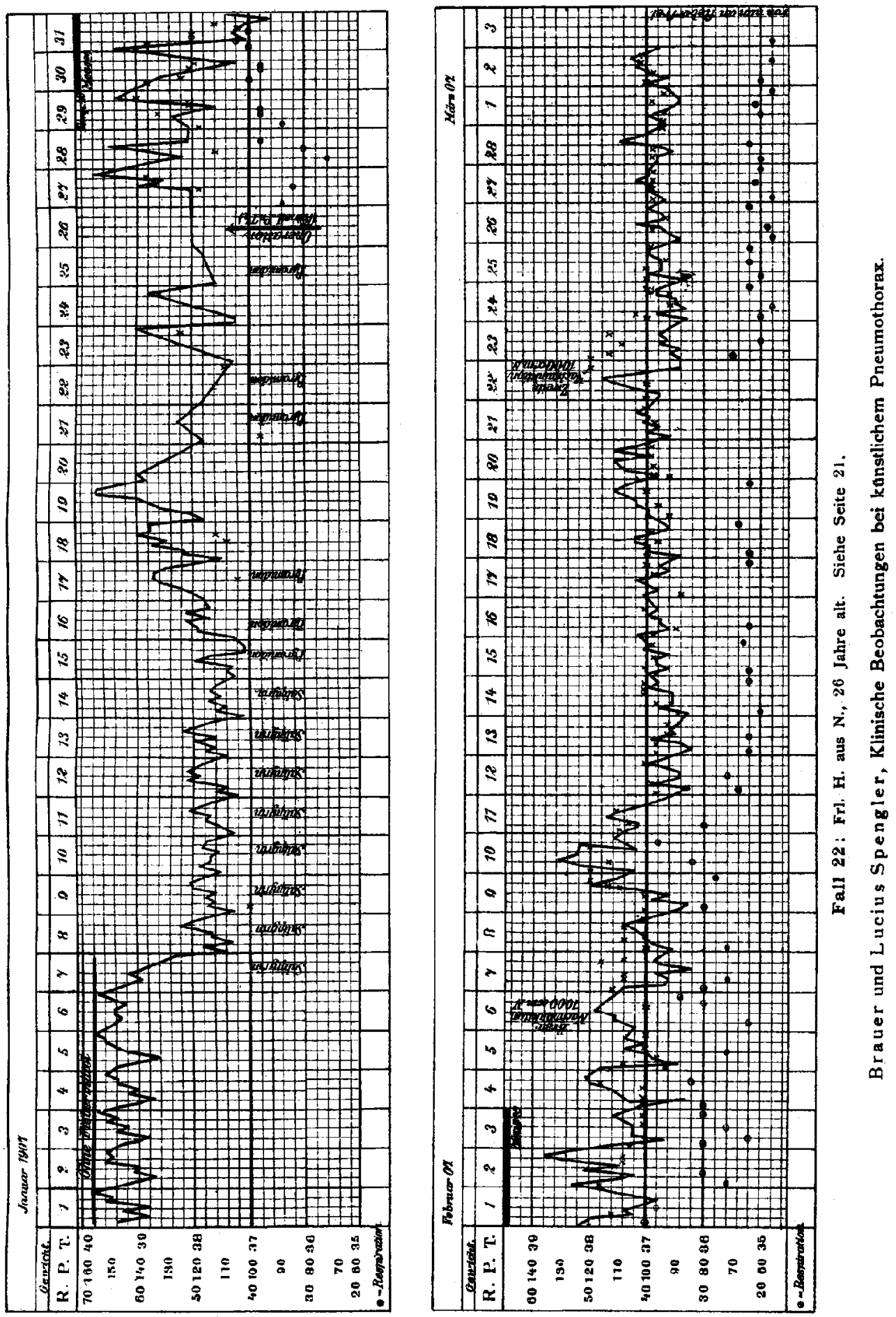

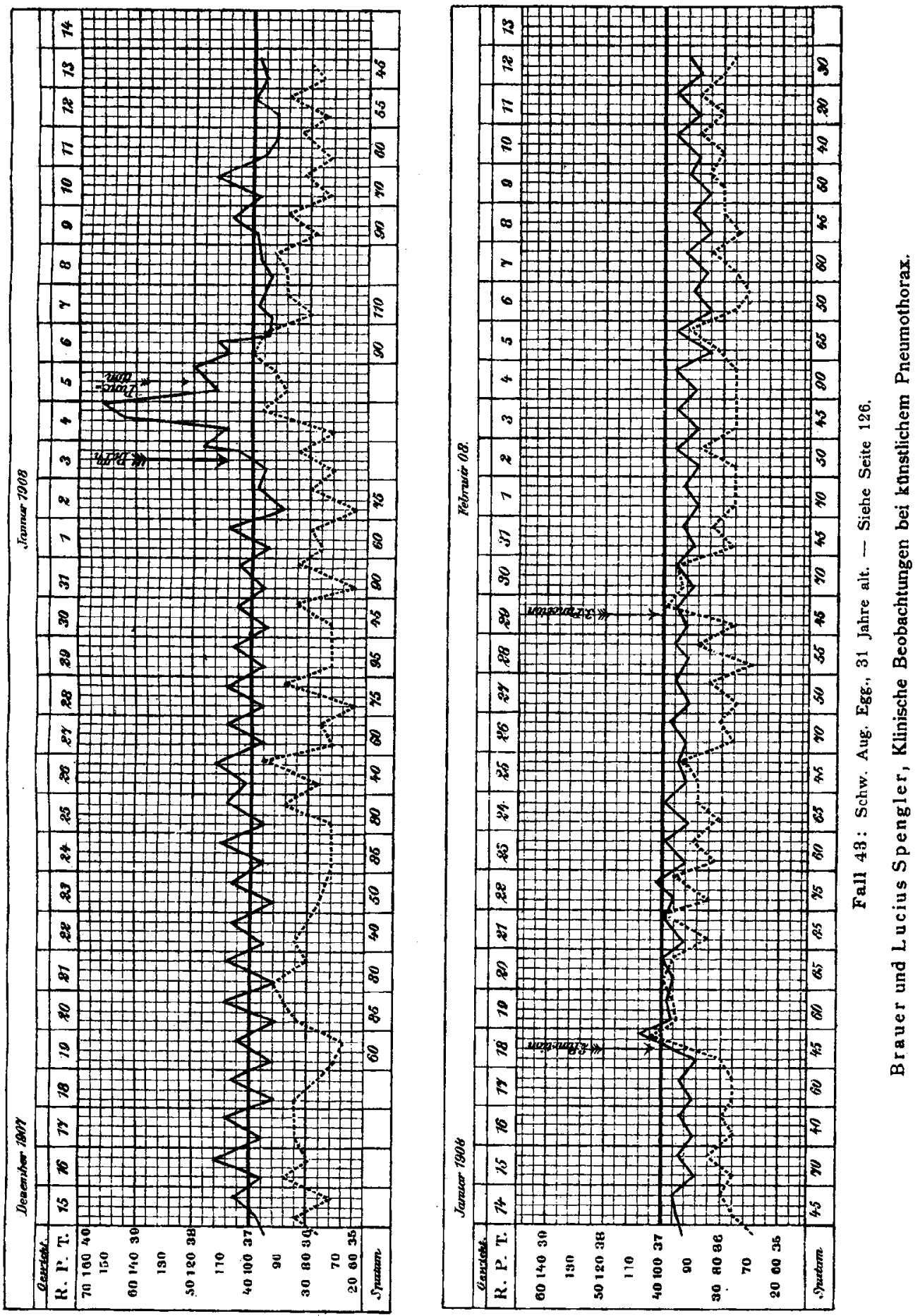

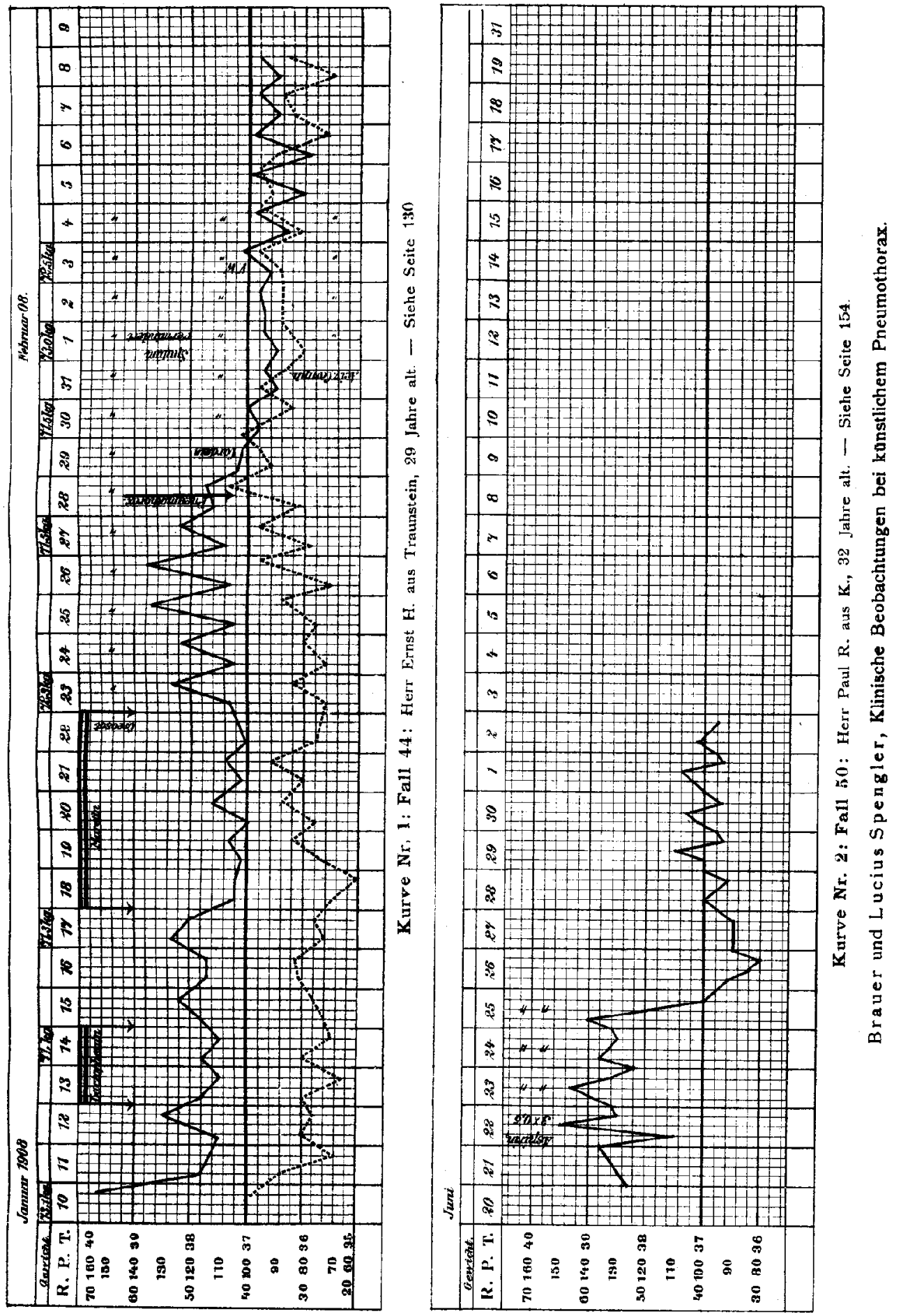


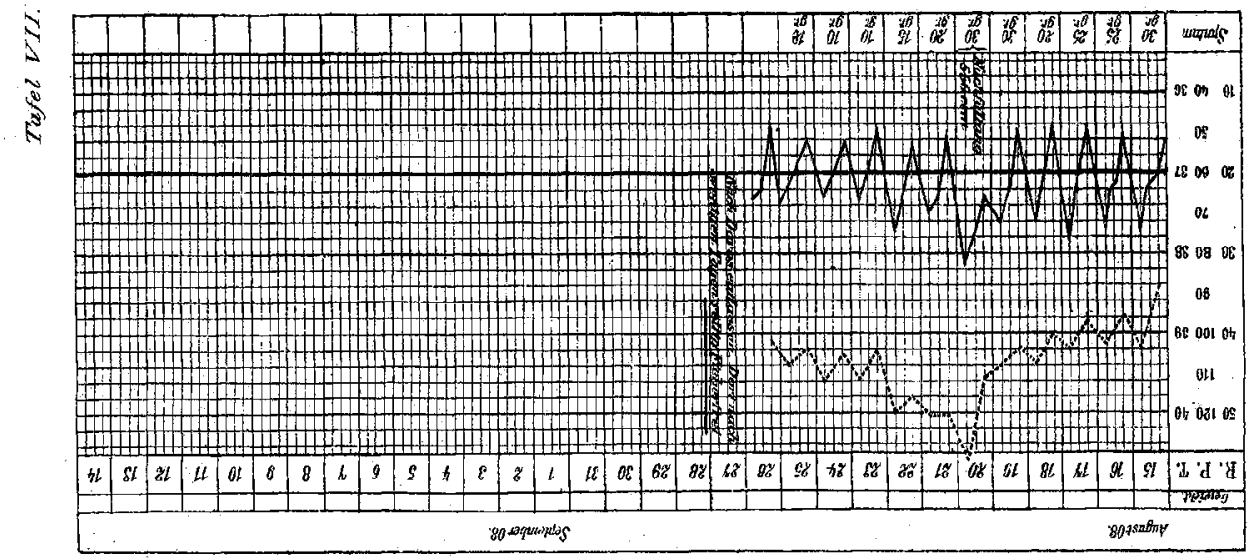

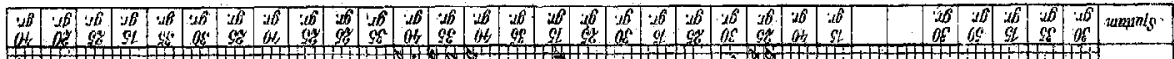

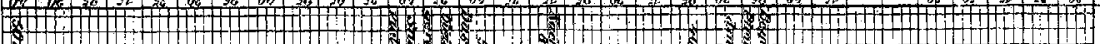

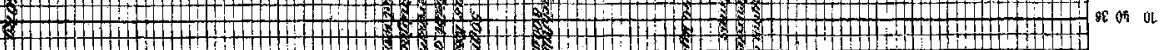

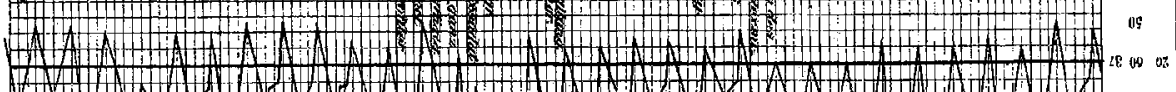
(2) A

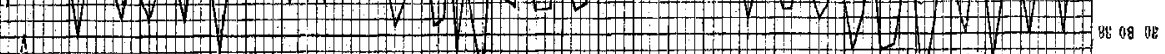
次

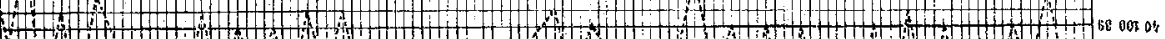

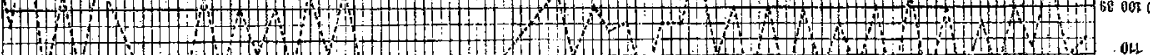
+13+1)

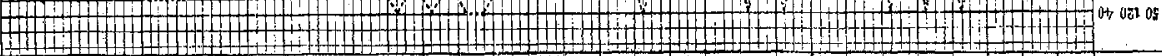

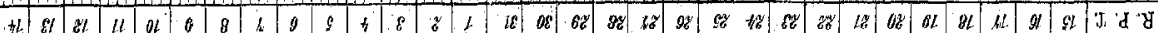
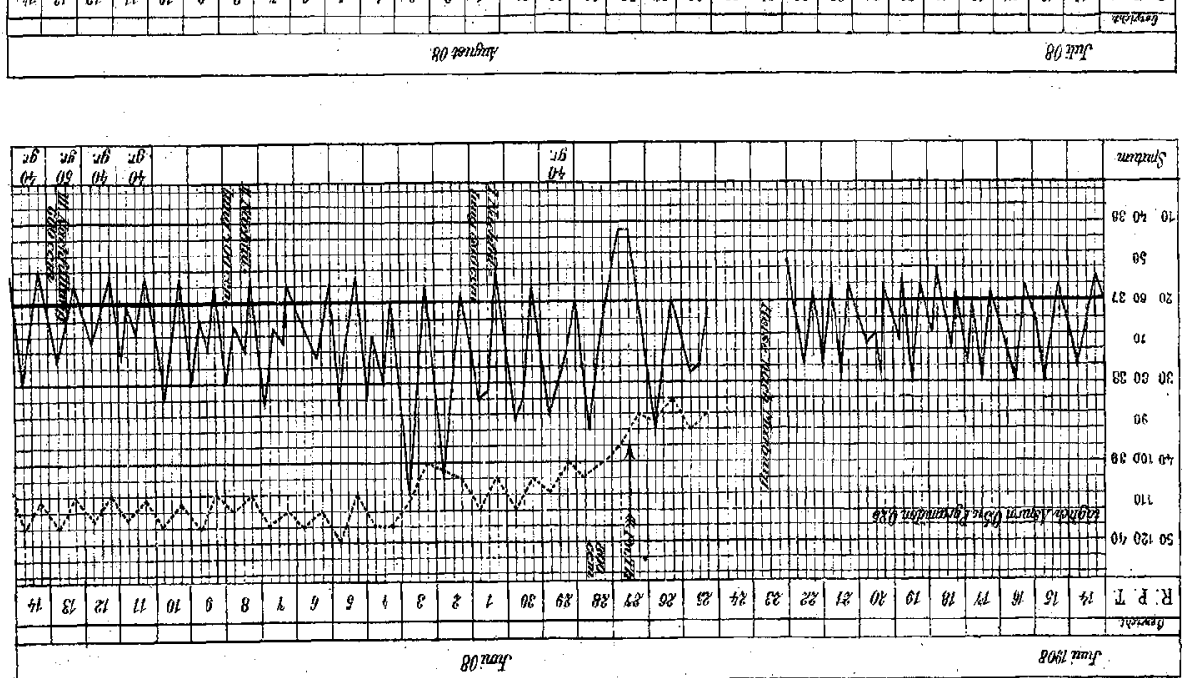
' 'в.

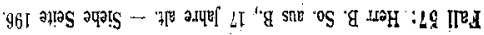
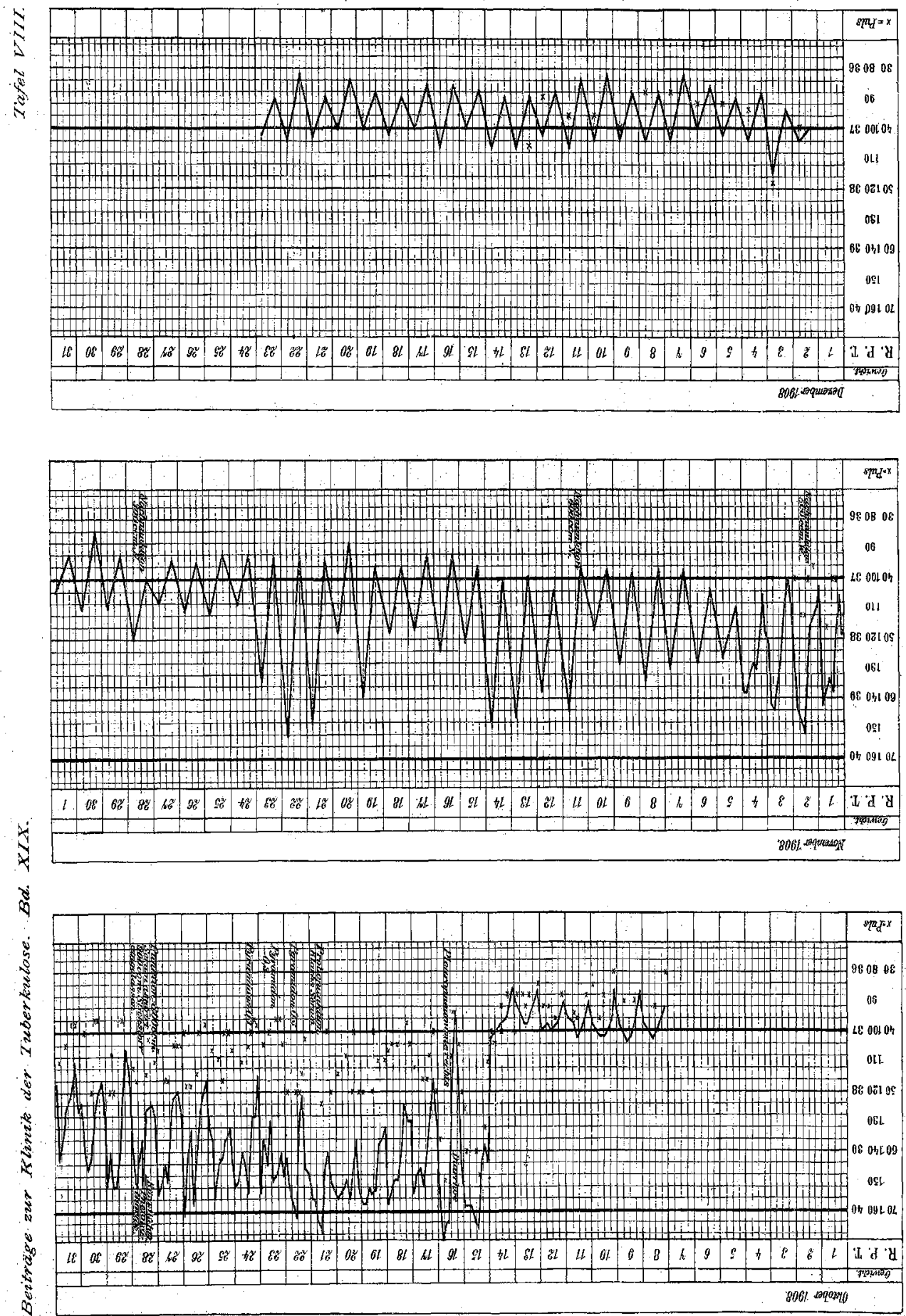

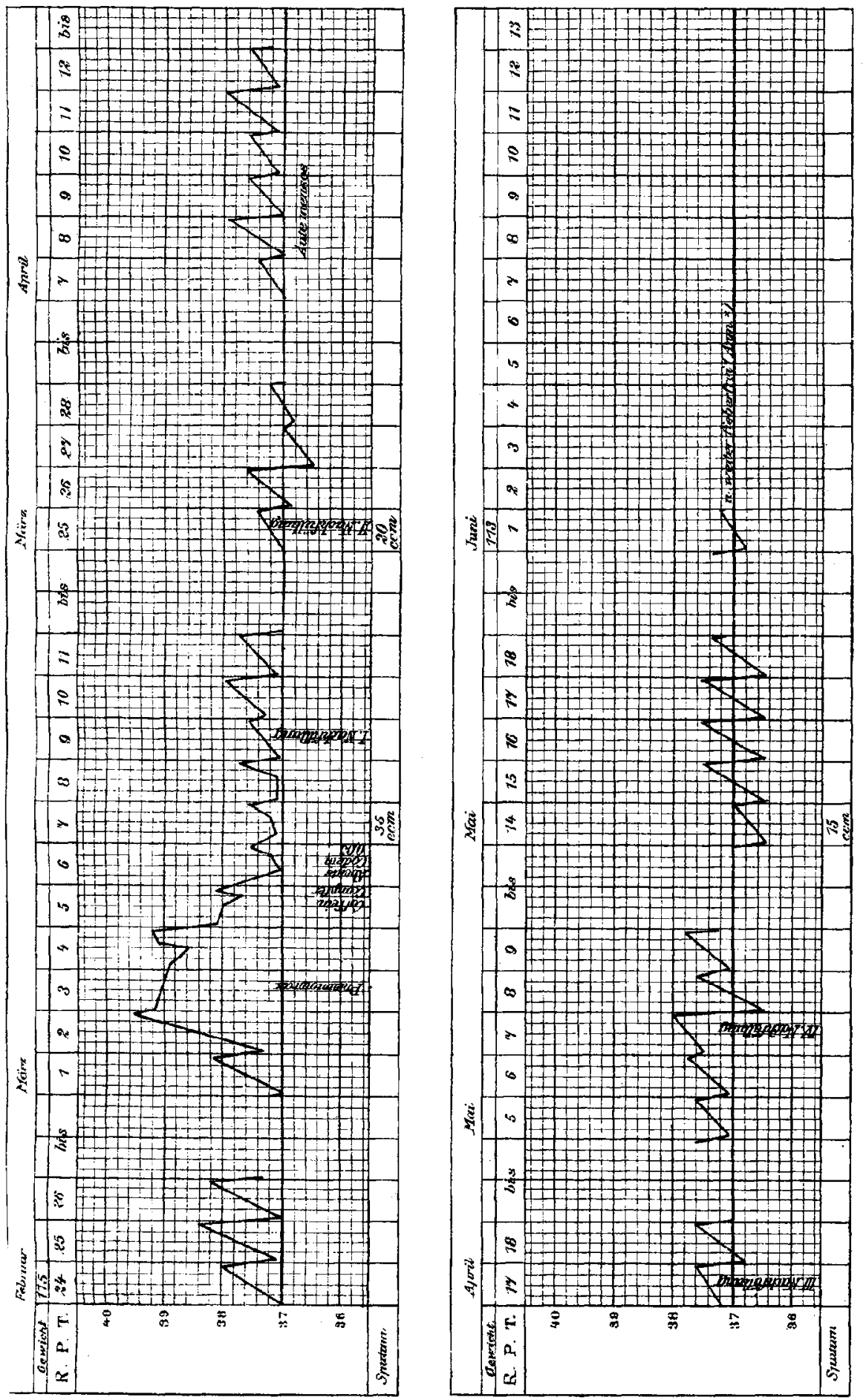

政

额

홀

政

空语

焉

要

당

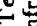

递

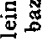

풍

政

昰

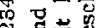

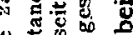

要

둥

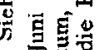

go

范苔的

更

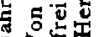

용

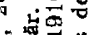

is

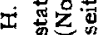

$\dot{E} \overline{\mathrm{z}} \mathbf{\mathrm { g }}$

4.

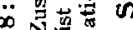

N小空。

$\Rightarrow$ 包官흠

का

政

돌

क

需

8 은

这

㱐

造

芒

든

ฮี

题焉 

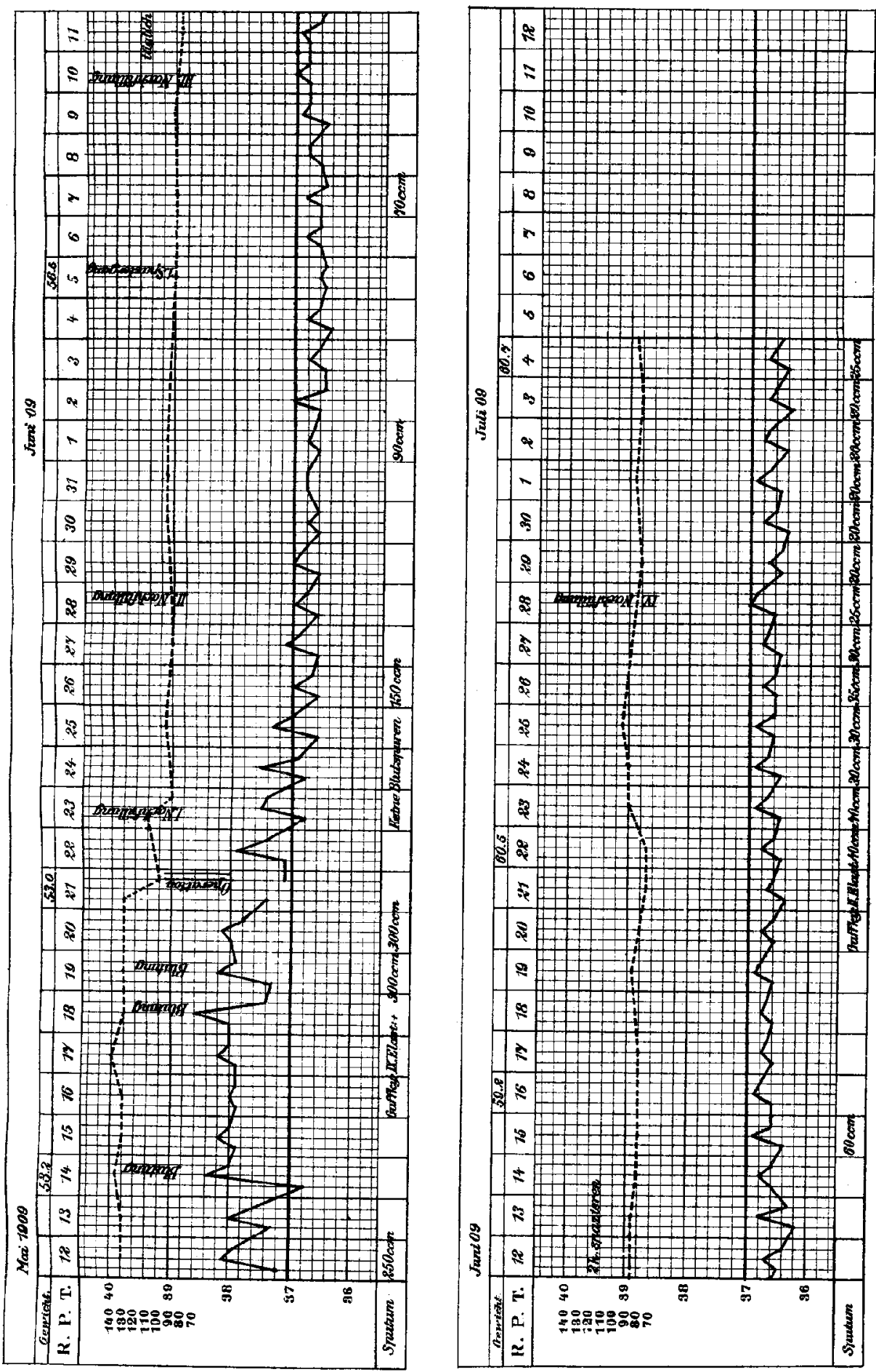

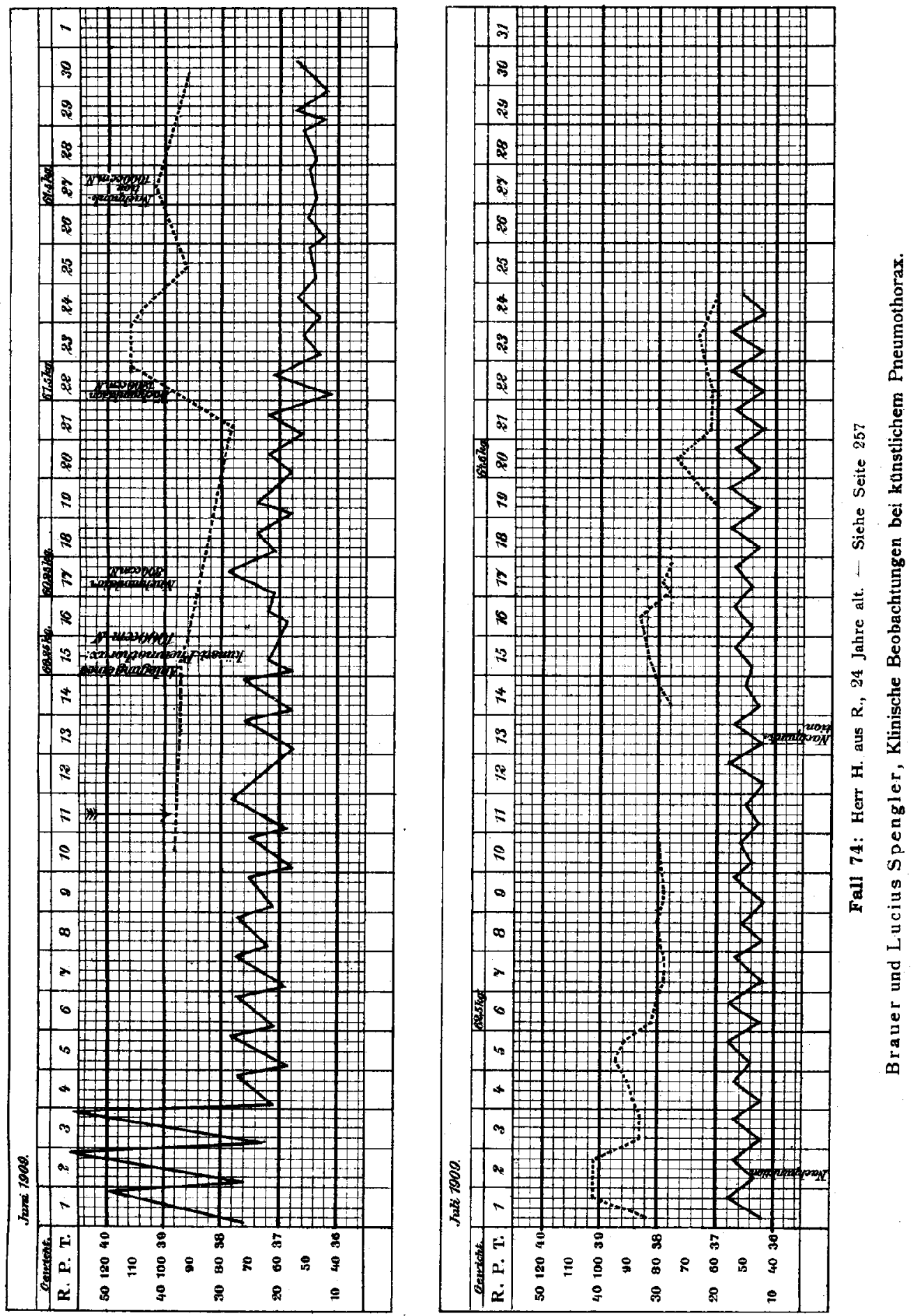

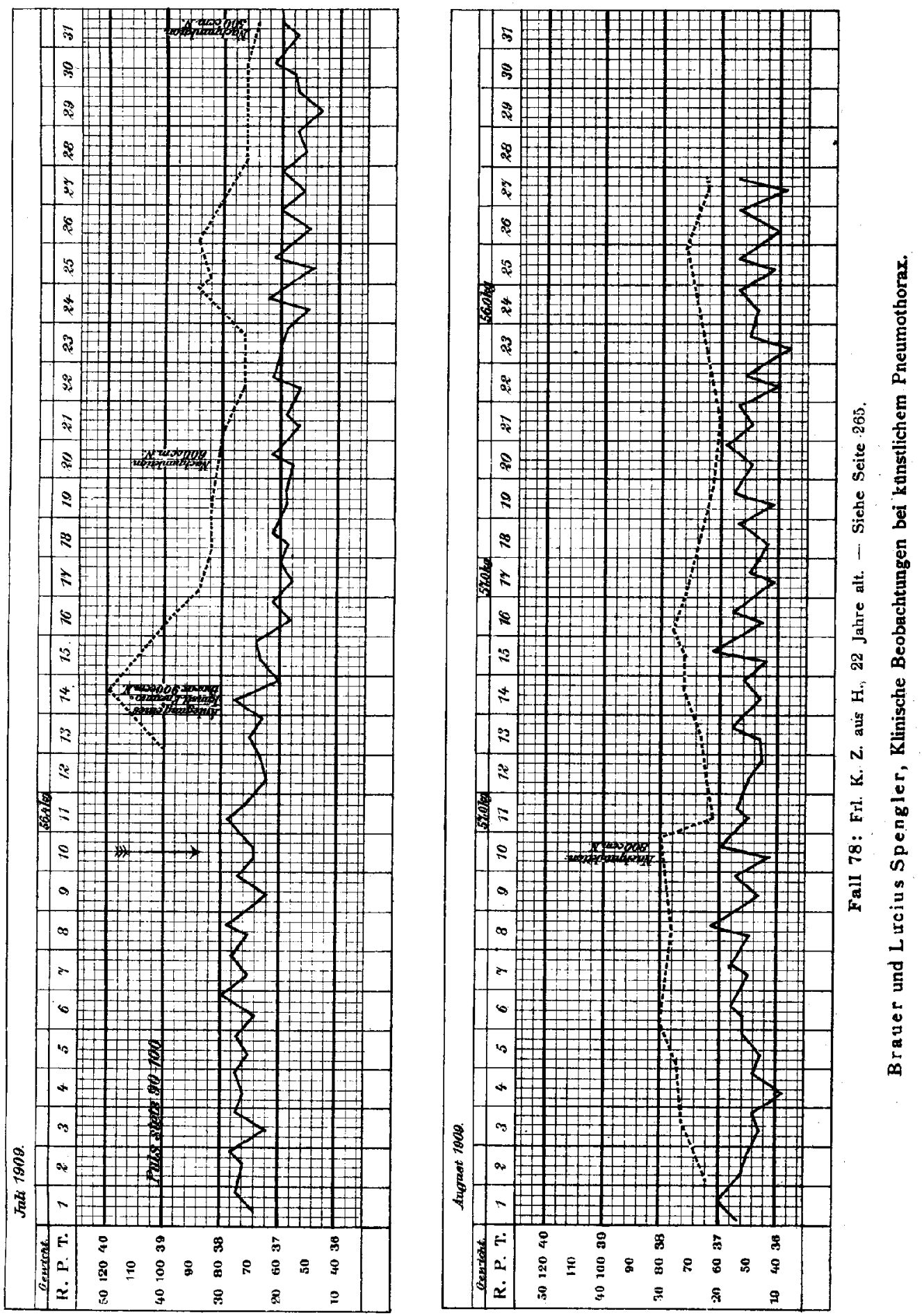